\title{
Selection from read-only memory with limited workspace th
}

\author{
Amr Elmasrya ${ }^{\mathrm{a}}$, Daniel Dahl Juhl ${ }^{\mathrm{b}}$, Jyrki Katajainen ${ }^{\mathrm{b}}$, Srinivasa Rao Sattic ${ }^{\mathrm{c}, 1}$ \\ ${ }^{a}$ Department of Computer Engineering and Systems, Alexandria University, Egypt \\ ${ }^{b}$ Department of Computer Science, University of Copenhagen, Denmark \\ ${ }^{c}$ School of Computer Science and Engineering, Seoul National University, South Korea
}

\begin{abstract}
Given an unordered array of $N$ elements drawn from a totally ordered set and an integer $k$ in the range from 1 to $N$, in the classic selection problem the task is to find the $k$-th smallest element in the array. We study the complexity of this problem in the space-restricted random-access model: The input array is stored on read-only memory, and the algorithm has access to a limited amount of workspace. We prove that the linear-time prune-and-search algorithmpresented in most textbooks on algorithms - can be modified to use $\Theta(N)$ bits instead of $\Theta(N)$ words of extra space. Prior to our work, the best known algorithm by Frederickson could perform the task with $\Theta(N)$ bits of extra space in $O\left(N \lg ^{*} N\right)$ time. Our result separates the space-restricted random-access model and the multi-pass streaming model, since we can surpass the $\Omega\left(N \lg ^{*} N\right)$ lower bound known for the latter model. We also generalize our algorithm for the case when the size of the workspace is $\Theta(S)$ bits, where $\lg ^{3} N \leq S \leq N$. The running time of our generalized algorithm is $O\left(N \lg ^{*}(N / S)+N(\lg N) / \lg S\right)$, slightly improving over the $O\left(N \lg ^{*}(N(\lg N) / S)+N(\lg N) / \lg S\right)$ bound of Frederickson's algorithm. To obtain the improvements mentioned above, we developed a new data structure, called the wavelet stack, that we use for repeated pruning. We expect the wavelet stack to be a useful tool in other applications as well.
\end{abstract}

Keywords: Selection algorithm, read-only memory, random-access machine, multi-pass streaming, bit vector, wavelet stack

The results of this paper were presented in preliminary form at the 19th Annual International Computing and Combinatorics Conference held in Hangzhou, China, in June 2013.

Email addresses: elmasry@alexu.edu.eg (Amr Elmasry), juhl.daniel@gmail.com (Daniel Dahl Juhl), jyrki@di.ku.dk (Jyrki Katajainen), ssrao@cse.snu.ac.kr (Srinivasa Rao Satti)

${ }^{1}$ The research of this author was partly supported by Basic Science Research Program through the National Research Foundation of Korea (NRF) funded by the Ministry of Education, Science and Technology (grant number 2012-0008241). 


\section{Introduction}

Let $\left\langle x_{1}, x_{2}, \ldots, x_{N}\right\rangle$ be a sequence of unordered elements, from a totally ordered set, stored in an array. Given an integer $k$ in the range $1 \leq k \leq N$, in the selection problem we want to find the $k$-th smallest of these elements. Without loss of generality, we can assume that the elements are distinct (since in the case of equal elements, the indices can be used to distinguish the elements). That is, the output will be a single index $j$ with a guarantee that $k-1$ elements are smaller than $x_{j}$ and $N-k$ elements are larger than $x_{j}$.

The asymptotic time complexity of the selection problem was settled to be $\Theta(N)$ by Blum et al. 3] in their celebrated article from 1973. Here we study the problem in the space-restricted random-access model, where the input elements are given in a read-only array and a limited amount of additional workspace is available. We first focus on the case where the amount of workspace is $\Theta(N)$ bits, and subsequently consider the general case with a more limited workspace. Surprisingly, although the time-space trade-offs for selection in this read-only space-restricted setting have been studied in several papers [4, 10, 16, 17, 21], its exact complexity is still not fully resolved (even after our study).

We start by describing an algorithm that solves the selection problem using $O\left(\lg ^{2} N\right)$ extra bits (or a logarithmic number of machine words). Although this algorithm does not match the time complexity of the best known algorithm using this amount of workspace, it illustrates some of the difficulties involved in designing algorithms for this model of computation, and also describes a few techniques that we shall use in our main algorithm. As in many other selection algorithms, we maintain two indices that specify the so-called filters. The elements whose values fall within the range of the two filters are still possible candidates for being the $k$-th smallest element. We say that the elements within the range of the filters are active. At the beginning of the algorithm, we scan the input and initialize the two filters to be the minimum and maximum elements. If $k=1$ or $N$, we are done; otherwise, all elements are active except the filters.

The algorithm proceeds recursively. Each recursive call takes the following parameters: (i) a contiguous segment of $M$ elements, specified by its first and last array indices, (ii) a pair of filters, specified by their positions in the input array, and (iii) a parameter $k$; and returns the $k$-th smallest element among the active elements within the segment. The recursive call proceeds by dividing the segment into $t$ zones of contiguous elements: each zone except the last constitutes $\lceil M / t\rceil$ elements, and the remaining elements form the last zone. We then check which of the $t$ zones contains the largest number of active elements (this idea is from [17]); we say that this zone is heavy. Next, we select the median of the heavy zone recursively. Let $x_{i 1}$ and $x_{i 2}$ be the filters (before the recursive calls) and $x_{m}$ the median found. After this first recursive call, we scan through the elements in the current segment to determine whether the $k$-th smallest element is in the interval $\left(x_{i 1} \ldots x_{m}\right)$, is equal to $x_{m}$, or is in the interval $\left(x_{m} \ldots x_{i 2}\right)$. If $x_{m}$ is the $k$-th smallest element, we return $x_{m}$ as output. In the other two cases, we update the filters. In the last case, we set $k$ to $k-\alpha$ if $\alpha$ smaller active elements were eliminated. Since $x_{m}$ is the median of the active 
elements in the heavy zone, at least $\frac{1}{2 t}$-th of the active elements will be removed from further consideration. Finally, we perform the second recursive call to find the $k$-th smallest of the remaining active elements in the whole segment.

For each subproblem we store: the segment boundaries, the present filters, and a bit indicating which of the recursive calls - the first or the second - is invoked. When $t$ is a constant, the maximum depth of the recursion stack is $O(\lg N)$. Thus, the overall workspace used is $O\left(\lg ^{2} N\right)$ bits.

We use $n$ to denote the number of active elements, and $M$ to denote the size of the contiguous segment where the elements reside. The worst-case running time can be described using the recurrence:

$$
T(n, M) \leq\left\{\begin{array}{l}
c_{1} \cdot M \text { if } n<n_{0} \\
T\left(n^{\prime},\lceil M / t\rceil\right)+T\left(n^{\prime \prime}, M\right)+c_{2} \cdot M \text { if } n \geq n_{0},
\end{array}\right.
$$

where $c_{1}, c_{2}$ and $n_{0}$ are positive constants, and $n^{\prime}$ and $n^{\prime \prime}$ denote the number of active elements of the subproblems in the first and second recursive calls, respectively. Since $n / t \leq n^{\prime} \leq n$, let $n^{\prime}=r \cdot n$ where $1 / t \leq r \leq 1$. It follows that $n^{\prime \prime} \leq(1-r / 2) \cdot n$. When we set $t=16$, for the case $n \geq n_{0}$ where $n_{0}$ is big enough, one can show by substitution that $T(n, M) \leq c \cdot(\sqrt{n}-1) \cdot M$ for a positive constant $c$ big enough compared to $c_{1}$ and $c_{2}$. Indeed, from the induction hypothesis, $T\left(n^{\prime},\lceil M / 16\rceil\right) \leq c \cdot(\sqrt{r \cdot n}-1) \cdot\lceil M / 16\rceil$ and $T\left(n^{\prime \prime}, M\right) \leq$ $c \cdot(\sqrt{(1-r / 2) \cdot n}-1) \cdot M$. Since $\sqrt{1-r / 2}<1-r / 4$, thus $\sqrt{r} / 16+\sqrt{1-r / 2}<1$ when $1 / 16 \leq r \leq 1$, and the induction hypothesis is validated. For these settings, the running time of the algorithm is therefore $O\left(N^{3 / 2}\right)$.

The above algorithm highlights several aspects that are important for algorithms designed for the space-bounded random-access machine. Since we cannot move or modify elements, neither can we utilize enough space to store the indices of the active elements, we have to scan the read-only array several times and pass over already eliminated elements. Due to the limited memory resources, we cannot store everything, and sometimes have to recompute information that has already been computed previously. Also because of the limited workspace, it might be necessary to resort to some bit tricks to save space and time.

The performance of the best known selection algorithms is summarized in Table 1 for workspace sizes specified as a function of the number of elements. In this paper we improve the known results when the amount of extra space is $\Theta(N)$ bits, by giving a new implementation for an adapted version of the algorithm of Blum et al. 3] that also runs in $\Theta(N)$ time. For the general case of $\Theta(S)$ bits of workspace, the best known algorithm is that of Frederickson 10. The running time of Frederickson's algorithm is $O\left(N \lg ^{*}(N(\lg N) / S)+N(\lg N) / \lg S\right)$ when $S=\Omega\left(\lg ^{3} N\right)$. We generalize our algorithm to run in $O\left(N \lg ^{*}(N / S)+\right.$ $N(\lg N) / \lg S)$ time and use $\Theta(S)$ bits, for any $\lg ^{3} N \leq S \leq N$, and thus improve Frederickson's algorithm (for a narrow range of $S$ ).

In the literature two main models of computation have been considered when handling read-only data: the multi-pass streaming model [4, 10, 16] and the space-restricted random-access model (that is used in this paper) [10, 17, 21]. The essential difference is that, in the streaming model, the read-only input 
Table 1: The best known algorithms for selecting the $k$-th smallest of $N$ elements in the space-restricted random-access model; $N$ is the number of elements of the read-only input and $k$ is an arbitrary integer between 1 and $N$. The algorithms (except that in [3]) work for a larger range of workspace, but we give their running times only for these specific values.

\begin{tabular}{|l|c|c|}
\hline \multicolumn{1}{|c|}{ Inventors } & Workspace in bits & Running time \\
\hline Munro and Raman [17] & $\Theta(\lg N)$ & $O\left(N^{1+\varepsilon}\right)$ \\
Raman and Ramnath [21] & $\Theta\left(\lg ^{2} N\right)$ & $O\left(N \lg ^{2} N\right)$ \\
Frederickson [0] & $\Theta\left(\lg ^{3} N\right)$ & $O(N \lg N / \lg \lg N)$ \\
Frederickson [10] & $\Theta(N)$ & $O(N \lg N)$ \\
Blum et al. [3] & $\Theta(N \lg N)$ & $\Theta(N)$ \\
\hline Elmasry et al. [this paper] & $\Theta(N)$ & $\Theta(N)$ \\
\hline
\end{tabular}

must be accessed sequentially but multiple scans of the entire input are allowed; in addition to the running time, the number of scans performed would be an optimization target. Chan [4] proved that Frederickson's algorithm is asymptotically optimal for the selection problem in the multi-pass streaming model. He questioned whether this lower bound would also hold in the space-restricted random-access model. We answer this question negatively, by surpassing the bound on the space-restricted random-access machine.

Our algorithms rely heavily on the random-access capabilities. The kernel of our construction is the wavelet stack - a new data structure that allows us to eliminate elements while being able to sequentially scan the active elements and skip over the eliminated ones. This data structure only requires a constant number of bits per element (instead of the usual $\lceil\lg N\rceil$ bits required for storing indices). The wavelet stack is by no means restricted to this particular application. We believe it would be generally useful for prune-and-search algorithms in the space-bounded setting. A wavelet stack comprises several layers of bit vectors, each supporting rank and select queries in $O(1)$ worst-case time [8, 13, 15, 20. Using the rank and select operations, we can navigate between the layers of the stack and perform successor queries efficiently.

\section{Previous results}

In this section we recall the main ideas of Munro-Paterson and Frederickson selection algorithms, some of which we shall use later. We also discuss the time-space lower bound for the selection problem in the multi-pass streaming model. In addition, we highlight some selection algorithms for strictly limited workspace and recent developments for integer data. Throughout this section we assume the available workspace to be $\Theta\left(s_{w}\right)$ words, i.e. $S=s_{w} \lg N$ bits.

\subsection{Munro-Paterson selection algorithm}

Munro and Paterson [16] outlined a selection algorithm for the multi-pass streaming model that achieves a running time of $O\left(N \lg s_{w}+N(\lg N) / \lg s_{w}\right)$, when $s_{w}=\Omega\left(\lg ^{2} N\right)$. It should be noted that this algorithm is originally designed to optimize the number of passes made over the input. 
The main idea of the algorithm is to repeatedly select two filter elements of improving quality. The filters determine which elements are still to be considered. Any element falling outside the range of the filters is simply ignored. After a number of iterations, there are few enough candidates remaining in the range of the filters so that we can find the designated element using a standard linear-time selection algorithm [3] within the limits of the available workspace.

In each pass a sample is constructed from the elements falling between the filters of the previous pass. An $s$-sample at level- $i$ is a sorted sequence of $s$ elements deterministically chosen from a population of $s \cdot 2^{i}$ candidates. A level-0 sample consists of $s=2\left\lceil s_{w} /(2 \lg N)\right\rceil$ candidates in sorted order, and is obtained by resuming the sequential scan of the input array. A level- $i s$-sample is a thinning of two $s$-samples from level- $(i-1)$, and is obtained by selecting every other element from each sample and then merging the two thinned samples. To utilize the storage efficiently, a bottom-up approach is employed to iteratively construct next-level samples once two are ready at a level. Thus, when an $s$ sample is constructed, there is at most one $s$-sample at each level other than the one that has just been produced. Let $n$ be the number of active elements at the beginning of a pass. At the end of that pass, two improved filters are selected from the $s$-sample at level $r=\lceil\lg (n / s)\rceil$. The ranks of the two new filters with respect to the sorted sample at level $r$ are $\left\lceil k / 2^{r}\right\rceil-r$ and $\left\lceil k / 2^{r}\right\rceil$.

The total running time of the Munro-Paterson algorithm is $O(N \lg s+$ $N(\lg N) / \lg s)$. The actual running-time analysis of the algorithm is due to Frederickson [10], whose arguments can be summarized as follows:

- Starting with $n$ active elements remaining in the range of two filters, the next pass will reduce the number of active elements to $O((n / s) \cdot \lg (n / s))$.

- The number of passes performed by the algorithm is $O((\lg N) / \lg s)$.

- Except for $\Theta(N)$ work done per pass to scan and compare the elements, the $O(N \lg s)$ time consumed in sorting the level-0 samples during the first pass dominates the rest of the work.

\subsection{Frederickson's improved selection algorithm}

As mentioned above, Frederickson [10] observed that the bottleneck of the Munro-Paterson algorithm is the sorting done to create the $s$-samples at level 0 during the first pass. Since there are $N / s$ such samples, their sorting cost accounts for the $N \lg s_{w}$ term in the running time.

Frederickson improved the algorithm by modifying the sampling procedure. Using a parameter $d$, the algorithm finds the $d$-quantiles of the size- $s$ sets that are gathered at level 0 (instead of sorting the sets). The execution of the algorithm can be divided into $\mathcal{P}$ phases, where $\mathcal{P}=\lg ^{*} s-2$. In each phase, the algorithm performs a constant number of passes until the number of elements is reduced to $N / \lg ^{(\mathcal{P})} s$. The value of $\mathcal{P}$ is decremented and a new phase is performed, repeating until $\mathcal{P}=0$. After each phase, the parameter is adjusted to $d=\lg ^{(\mathcal{P})} s$. As $\mathcal{P}$ decreases by one in each phase, $d$ increases exponentially. Each pass requires $O(N+n \lg d)$ time, where $n$ is the number of active 
candidates before the pass. Initially, only a constant number of quantiles are computed, and as the number of remaining candidates decreases the number of quantiles computed per sample increases exponentially. At the low levels, instead of thinning and merging the $d$-samples, they are simply merged such that at level $i$ the samples have size $2^{i} \cdot d$. Once $2^{i} \cdot d \geq s$, the thinning and merging procedure is again in use; this keeps the sample size bounded by $s$. As before, the new filters are computed from a final level- $r$ sample (as in the Munro-Paterson algorithm). In all, the work done during each pass, and hence also during each phase, is $\Theta(N)$. The above procedure allows us to reduce the input size to $O(N / \lg s)$ once $d=s$, which happens after $O\left(\lg ^{*} s\right)$ phases. After that, the Munro-Paterson algorithm is back to action. Combining this outcome with that of the Munro-Paterson algorithm, the running time of the overall algorithm becomes $O\left(N \lg ^{*} s_{w}+N(\lg N) / \lg s_{w}\right)$ for $s_{w}=\Omega\left(\lg ^{2} N\right)$. The insights of the analysis can be summarized as follows:

- Starting with $n$ active elements remaining in the range of two filters, performing a phase that starts by finding the $d$-quantiles of size- $s$ sets, the number of active elements is reduced to $O((n / s) \cdot \lg (n / s)+n / d)$.

- After $O\left(\lg ^{*} s\right)$ phases, there are at most $N / \lg s$ elements remaining.

- Each phase runs in $\Theta(N)$ time.

Chan [4] noted that the running time can be improved to $O\left(N \lg ^{*}\left(N / s_{w}\right)+\right.$ $\left.N(\lg N) / \lg s_{w}\right)$, by simply switching to the Munro-Paterson algorithm once $d=\min (s, N / s)$ (instead of switching when $d=s$ ). The reader is encouraged to verify that this bound is better than the $O\left(N \lg ^{*} s_{w}+N(\lg N) / \lg s_{w}\right)$ bound.

\subsection{Selection algorithms for more limited workspace}

The selection algorithms presented up to this point require at least $s_{w}=$ $\Omega\left(\lg ^{2} N\right)$ words of workspace to be available. For $s_{w}=o(\lg N)$, Munro and Raman [17] developed an algorithm based on recursively finding the median of a block of candidates to filter the elements until the required element is found. Their algorithm runs in $O\left(2^{s_{w}} s_{w} ! N^{1+1 / s_{w}}\right)$ time. When $s_{w}=1 / \varepsilon=O(1)$, i.e. with constant number of words of workspace, the running time becomes $O\left(N^{1+\varepsilon}\right)$. It is also worth noting that the total number of comparisons made by their algorithm is minimized for $s_{w}=O(\sqrt{(\lg N) / \lg \lg N})$, which gives a running time of $O\left(N^{1+O}(\sqrt{(\lg \lg N) / \lg N})\right.$. Munro and Raman also proved that if the elements in the input are assumed to be in random order, then their algorithm can be modified to have an average-case running time of $O\left(N \lg \left((\lg N) / \lg s_{w}\right)\right)$. Chan 4 showed how this algorithm can be randomized so that the assumption on the order of elements in the input is not needed.

Raman and Ramnath 21 improved the performance when $s_{w}$ is $o\left(\lg ^{2} N\right)$ and $\Omega(\lg N)$, by describing an algorithm that finds a pair of approximate medians and uses them to construct a three-way partition of the active elements. The running time of this algorithm is $O\left(N \lg ^{2} N\right)$ when $s_{w}=\Theta(\lg N)$. They 
also presented a generalization of this algorithm for smaller values of $s_{w}$, by describing how to determine the desired approximate median-pair with less space. The running time of the modified algorithm is $O\left(s_{w} N^{1+1 / s_{w}} \lg N\right)$, which is an improvement over Munro and Raman's algorithm [17] when $s_{w}=O(\lg N)$ and $2^{s_{w}} s_{w}$ ! $>s_{w} \lg N$ (e.g. when $s_{w} \geq c \cdot(\lg \lg N) / \lg \lg \lg N$ for some positive constant $c$ ). Raman and Ramnath also described how the running time can be reduced further if more space is available. This is done by computing a set of three or more splitters instead of the pair of approximate medians, allowing the candidates to potentially be split into more than three buckets. The running time of this algorithm is $O\left(N \lg N+N\left(\lg ^{2} N\right) / \lg ^{2} \mathcal{I}\right)$ when $s_{w}=O\left(\mathcal{I}^{2}(\lg N) / \lg \mathcal{I}\right)$, where $\mathcal{I} \geq 2$ is an integer parameter. This is worse than Frederickson's algorithm by a factor of $(\lg N) / \lg \lg N$ when $s_{w}=\Theta\left(\lg ^{2} N\right)$, but unlike Frederickson's algorithm, it can be applied in cases where $s_{w}$ is $o\left(\lg ^{2} N\right)$ and $\Omega(\lg N)$.

\subsection{A lower bound in the multi-pass streaming model}

Munro and Paterson [16], using adversarial arguments, showed that any comparison-based selection algorithm in the multi-pass streaming model must perform $\Omega\left((\lg N) / \lg s_{w}\right)$ passes. Chan [4, also using adversarial arguments, proved that any deterministic comparison-based selection algorithm in this model must use either $\Omega(p)$ passes or $\Omega\left(N \lg ^{(p)}\left(N / s_{w}\right)\right)$ comparisons (for any $p$ ), indicating that any such algorithm must take $\Omega\left(N \lg ^{*}\left(N / s_{w}\right)\right)$ time. Combining the two results, it follows that any comparison-based deterministic selection algorithm for the multi-pass streaming model must spend $\Omega\left(N \lg ^{*}\left(N / s_{w}\right)+\right.$ $\left.N(\lg N) / \lg s_{w}\right)$ time on either scanning or comparisons. Chan 4 posed as an open problem whether this bound also holds for the space-restricted randomaccess model. We show that this is not the case, indicating that the two models are distinct in the context of deterministic selection.

\subsection{Integer selection}

Recent work by Chan, Munro and Raman [5] has indicated that faster selection algorithms are possible if we restrict the input elements to be a sequence of integers. When the input elements come from the universe $\{1,2, \ldots, U\}$, they presented two algorithms, one running in $O\left(N \lg _{s_{w}} U\right)$ using $O\left(s_{w}\right)$ words of space for any $s_{w}$ from 1 to $N$, the other running in $O\left(N(\lg N) \lg _{s_{w}} \lg U\right)$ while using $O\left(s_{w}\right)$ words of space for any $s_{w}$ from 1 to $\lg U$. The first algorithm determines the bits of the $k$-th element by iteratively counting the number of 1- and 0-bits among the candidates at the current bit location and comparing these counts with $k$. This approach only uses $s_{w}=O(1)$ words, but can be easily extended to handle $b$ bits during each pass when we have $s_{w}=2^{b}$ extra words of workspace available. The second algorithm is significantly more involved, but the basic idea is to utilize the bit-prefixes of the integers to efficiently select approximate medians that can then be used in a well-known prune-and-search approach for the selection problem. The first algorithm is a significant improvement over existing methods for small universe sizes, whereas the second algorithm is less sensitive to the universe size and thus provides an improvement over existing algorithms for a wider range of universe sizes. 


\section{Basic toolbox}

In this section we describe the basic tools used in our algorithms.

\subsection{Bit vectors with rank and select support}

A bit vector is an array of bits (0's and 1's). Consider a bit vector $V$ that supports the following operations:

$V$ access $(i)$ : Return the bit at index $i$, also denoted as $V[i]$.

$V \cdot \operatorname{rank}(i)$ : Return the number of 1-bits among the bits $V[1], V[2], \ldots, V[i]$.

$V \operatorname{select}(j)$ : Return the index of the $j$-th 1-bit, i.e. when the return value is $i$, $V[i]=1$ and $\operatorname{rank}(i)=j$.

On a word RAM with $w$-bit word size, one can store a sequence of $N$ bits using $\lceil N / w\rceil$ words, such that any substring of at most $w$ bits - not only a single bit - can be accessed in $O(1)$ worst-case time.

There exist several space-efficient solutions to support the above operations in $O(1)$ worst-case time. Jacobson 13 showed how to support rank and select in $O(\lg N)$ bit probes using $o(N)$ bits in addition to the bit vector. Clark and Munro [8, 15] showed how to support the queries in $O(1)$ worst-case time using $O(N / \lg \lg \lg N)$ bits of extra workspace on a RAM with word size $\Theta(\lg N)$ bits. Raman et al. 20 improved the space bound to $O(N(\lg \lg N) / \lg N)$ bits, which was shown by Golynski [1] to be optimal provided that the bit vector is stored in plain form (using $N$ bits or $\lceil N / w\rceil$ words). The basic idea of these solutions is to divide the input into blocks, store the rank and select values for some specific positions, and compute the rank and select values for the remaining positions on the fly using: the stored values, values in some precomputed tables, and bits in the bit vector under consideration.

Note that the requirements on the bit vectors for our use in this paper are that (i) the space usage must be $O(N)$ bits, (ii) the operations must have $O(1)$ worst-case cost, and (iii) the construction of the supporting structures must take $O(N)$ worst-case time. For these requirements, Chazelle [7] described a simple solution to support the rank operation. After breaking the bit vector into words, for the first bit of each word a landmark is computed that is the number of 1-bits preceding this position. Let the words be $B_{1}, B_{2}, \ldots, B_{\lceil N / w\rceil}$ and the landmarks be $L_{1}, L_{2}, \ldots, L_{\lceil N / w\rceil}$. To compute $\operatorname{rank}(i)$, we locate the corresponding word $B_{j}$ where $j=\lceil i / w\rceil$, and calculate the offset $f$ within this word as $f=i-w \cdot\lfloor i / w\rfloor$. Then we mask the bits up to index $f$ in $B_{j}$ and calculate the number of 1-bits in the masked part; let this number be $q$. As the end result, we output $L_{j}+q$ as $\operatorname{rank}(i)$. The only remaining part is how to calculate the number of 1-bits in a word, but this can be done in $O(1)$ time using precomputed tables of size $o(N)$ bits. (In practice, one can use the populationcount function that is a hardware primitive in most modern processors.)

Let $Q$ be the number of ones in the bit vector $V$. To support the select operations, we construct an array of length $\lfloor Q / \lg N\rfloor$, using $O(N)$ bits, whose 
$j$-th entry stores the position of the $(j\lceil\lg N\rceil)$-th one in $V$, for $1 \leq j \leq\lfloor Q / \lg N\rfloor$. If the difference between two consecutive entries in this array is at least $\lg ^{2} N$, then we store the positions of all the $\lceil\lg N\rceil$ ones in between the two positions using $O\left(\lg ^{2} N\right)$ bits. If the difference between two consecutive entries is less than $\lg ^{2} N$, we construct a complete tree with branching factor $\sqrt{\lg N}$ and constant height that stores the bit sequence between the two positions at its leaves, such that select queries in this range can be answered in constant time. See [20] for the details of such a structure.

\subsection{Wavelet stacks}

In a prune-and-search algorithm, where some of the answer candidates are repeatedly eliminated, the set of active elements can be compactly represented using a bit vector. The history of the decisions made by such an algorithm can be conveniently described using a stack of bit vectors. We call this kind of data structure a wavelet stack because of its resemblance to a wavelet tree [12, 18. In the rest of this section, we describe the wavelet-stack data structure in detail. In the next section, we show how it can be used to solve the selection problem. We believe that this data structure will be useful in other applications as well.

Let $\left\langle x_{1}, x_{2}, \ldots, x_{N}\right\rangle$ be a sequence of $N$ elements given in a read-only array. Assume we want to find a specific subset of these elements using prune-andsearch elimination. A prune-and-search algorithm is a recursive procedure that may call itself several times. Hence, we need a recursion stack to keep track of the subproblems being solved. In addition to a recursion stack (with constantsize activation records), we maintain a stack of bit vectors to mark the active elements in the current configuration. The $i$-th bit in the bit vector at a given level corresponds to the $i$-th active element (in the left-to-right order) at the level below, and a 1-bit in a bit vector at a level indicates that the corresponding element is still active up to the current level. Using rank and select operations on the bit vectors, we can scan the active elements at any level (and avoid scanning the pruned elements) faster than a left-to-right scan of the input array.

In an abstract form, the wavelet stack is a stack of bit vectors, $H$, that can efficiently answer two types of queries:

H.active? $(i)$ : Return whether the element $x_{i}$ is active at the current configuration.

H.index $(j)$ : Return the index of the $j$-th active element, i.e. the index of the element corresponding to the $j$-th 1-bit of the top-most bit vector.

To fully understand these operations, we have to consider a concrete implementation of a wavelet stack (see Fig. 1). A wavelet stack is a hierarchy of bit vectors. The bottom-most level stores one bit per element, since at the beginning all elements are potential answers (i.e. active). If we have $y$ 1-bits at level $\ell$, the bit vector at level $\ell+1$ is of size $y$. Therefore, the bit vectors become smaller and smaller as we eliminate more elements from further consideration.

The two operations have a nice symmetry: the operation active? can be supported by traversing up from the bottom to the top of the stack, and the 


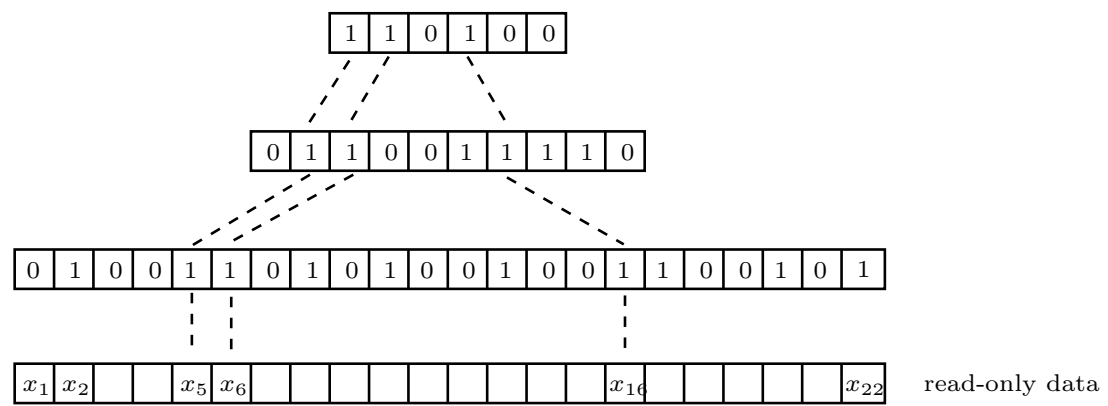

Figure 1: A wavelet stack for an array of 22 elements. Only elements $x_{5}, x_{6}$, and $x_{16}$ are active at the top-most level.

operation index can be supported by traversing down from the top to the bottom of the stack. To implement active?(i), we compute $\operatorname{rank}(i)$ at the bottom-most level, which gives us the index to access in the bit vector immediately above. Continuing upwards and relying on rank, we either reach a level where the bit corresponding to the index value is 0 , indicating that the element $x_{i}$ is not active any more, or reach the top-most level where the bit value is 1 , indicating that $x_{i}$ is still active. To implement index $(j)$, i.e. to return the $j$-th active element at the top-most level, we start from the top-most level and compute $\operatorname{select}(j)$. Then we use the returned index at the level immediately below. This way, we can proceed down using select until we reach the bottom-most level. The index returned at this level is the index of the $j$-th active element in the input array.

We can summarize the performance of the data structure as follows:

Theorem 3.1. Assume that we have built a wavelet stack of height $h$ for a read-only array of $N$ elements. Furthermore, assume that at each level we have succeeded in eliminating a constant fraction of the elements.

1. The data structure requires $\Theta(N)$ bits in total.

2. The total time used in the construction of the data structure is $\Theta(N)$.

3. Both active? and index operations take $O(h)$ worst-case time.

Proof: Since the number of bits needed at each level is only a constant fraction of that needed at the level below, for some constant $c<1$, the total number of bits of all the bit vectors is bounded by $\sum_{i=1}^{h} c^{i-1} N=\Theta(N)$ bits. The supporting structures for rank and select also sum to $O(N)$ bits. Since the length of the bit vectors is not known beforehand and since their sizes may vary, we can allocate a header storing references to a single bit vector that contains the bits stored at all levels together. This header will only require $O(h \lg N)=O\left(\lg ^{2} N\right)$ bits.

The construction of a bit vector, including the supporting structures, can be done in time linear in the vector size. The construction time of the wavelet stack can also be expressed as a geometric series, and is thus $\Theta(N)$. Since the structure has $h$ levels, and the rank and select operations take $O(1)$ worst-case time at each level, it can support active? and index operations in $O(h)$ time. 


\section{Selection with $\Theta(N)$ bits}

In this section we show how to utilize the prune-and-search algorithm of Blum et al. 3. (also described in [9, Section 9.3]) such that it only requires $\Theta(N)$ bits of space - instead of $\Theta(N)$ words - but still runs in $\Theta(N)$ time.

The main idea of the algorithm is to select an element from the set of active elements, and use it to make the set of candidates smaller (by a constant factor). This is done repeatedly until the required element is found. In the variant considered here we use a wavelet stack to keep track of the decisions made by the algorithm. The $k$-th smallest among $n$ active elements is found as follows.

1. A new bit vector $V$ is pushed onto the top of the wavelet stack. The size of this bit vector equals the number of the currently active elements $n$.

2. Divide the sequence of $n$ elements into groups of size $\lceil N / \lg N\rceil$ in the same order as in the input array (the last group may have less elements). Find the median of each of the, at most, $\lceil\lg N\rceil$ groups, by holding the indices of the elements of each group in the available workspace and applying a standard linear-time selection algorithm [3].

3. Store the indices of the found medians in the available workspace. Find the median $x_{\mu}$ of the medians of the groups, applying a standard linear-time selection algorithm 3 .

4. Scan through the active elements and count the number $\sigma$ of those smaller than $x_{\mu}$. If $k=\sigma+1$, stop and return $\mu$ as an answer. If $k \leq \sigma$, mark the elements smaller than $x_{\mu}$ as the only active elements in $V$ and recursively compute the $k$-th smallest of these elements. Otherwise, if $k>\sigma+1$, mark the elements larger than $x_{\mu}$ as the only active elements in $V$ and set $k$ to $k-\sigma-1$ before the recursive call.

5. When $n$ is at most $\lceil N / \lg N\rceil$, copy the indices of the active elements into the available workspace (releasing the space used by the wavelet stack), and find the $k$-th smallest element using a standard linear-time selection algorithm [3].

The analysis of this algorithm is almost identical to that of the original algorithm of Blum et al. 3. The key point is that, even though the input is in a read-only array, we do not waste time in browsing through the elements that have already been eliminated, as we rely on the rank-select operations supported by the bit vectors to scan through the active elements efficiently. The only overhead is that when we want to access an element we have to traverse down the wavelet stack.

The performance of the algorithm is summarized in the following theorem:

Theorem 4.1. The $k$-th smallest of $N$ elements in a read-only array can be found in $\Theta(N)$ time using $\Theta(N)$ extra bits in the worst case.

Proof: At step 2 of the algorithm, the number of elements of each group is at most $\lceil N / \lg N\rceil$. In accordance, the indices of all the elements of a group can be simultaneously stored using $O(N)$ bits of workspace. A standard linear-time 
selection algorithm can then be applied on each group at a time. Similarly, the median of medians can be found in linear time at step 3 of the algorithm within the storage limitations of the available workspace.

The main observation is that the number of elements pruned at step 4 of the algorithm is at least $n / 4-O(\lg N)$, where $n$ is the number of active elements before the pruning. Hence, the number of the surviving active elements before the next recursive call is at most $3 n / 4+O(\lg N)$. Since $n$ must be larger than $\lceil N / \lg N\rceil$ to perform a recursive call, thus the number of active elements before the $i$-th recursive call is at most $(3 / 4+\varepsilon)^{i-1} N$, where $\varepsilon$ is $o(1)$ compared to $N$. Following Theorem 3.1, the total size of all the bit vectors and the accompanying structures of the wavelet stack is $\Theta(N)$ bits and its construction time is $\Theta(N)$. To calculate the time for scanning over the active elements, we note that getting the successor of each active element at the $i$-th recursive call consumes $\Theta(i)$ time. It follows that the total time for scanning over the active elements in all the recursive calls is $\Theta\left(\sum_{i \geq 1} i \cdot(3 / 4+\varepsilon)^{i-1} \cdot N\right)=\Theta(N)$.

\section{Selection with $\Theta(S)$ bits}

In this section we extend our algorithm to handle the more general case of using a workspace of $\Theta(S)$ bits, where $\lg ^{3} N \leq S \leq N$. The main idea is to use Frederickson's algorithm [10 to prune the elements, and stop its execution when the number of active elements is at most $S$. To complete the selection process, we resume pruning using an $O(N)$-time algorithm that we present next.

We use the following lemma, which is based on Frederickson's algorithm discussed in Section 2.2. We refer the reader to [10] for the full details.

Lemma 5.1. The number of active elements can be reduced from $N$ to $S$ during the initial phases of Frederickson's algorithm in $O\left(N \lg ^{*}(N / S)\right)$ worst-case time, assuming $S=\Omega\left(\lg ^{3} N\right)$.

We now generalize our selection algorithm from the previous section to obtain time-space trade-offs. In particular, we describe a selection algorithm that takes $O\left(N \lg ^{*}(N / S)+N(\lg N) / \lg S\right)$ time given only $\Theta(S)$ bits of workspace, where $\lg ^{3} N \leq S \leq N$.

Recall that Frederickson's selection algorithm takes $O\left(N \lg ^{*}(N(\lg N) / S)+\right.$ $N(\lg N) / \lg S)$ time, for any $S=\Omega\left(\lg ^{3} N\right)$. If $S \leq \sqrt{N \lg N}$, we simply use Frederickson's algorithm all the way, and the resulting running time is as claimed $O\left(N \lg ^{*}(N(\lg N) / S)+N(\lg N) / \lg S\right)=O\left(N \lg ^{*}(N / S)+N(\lg N) / \lg S\right)$. From now on we assume that $S>\sqrt{N \lg N}$. We apply a trimmed execution of Frederickson's algorithm as specified in Lemma 5.1. The outcome is two filters that guard the, at most, $S$ active elements. Consequently, we are left with the task of selecting the designated element among those candidates.

Using a wavelet stack and a bit vector supporting rank and select queries, we can finish the pruning in $O(N)$ time. We divide the input sequence (consisting of $N$ elements) into $S$ buckets, where the $u$-th bucket consists of the elements from the input sequence with indices in the range $[(u-1) \cdot\lceil N / S\rceil+1 \ldots u \cdot\lceil N / S\rceil]$, 
for $1 \leq u \leq S$ (except possibly the last bucket). We create the count vector $C$ - a static bit vector that indicates the number of active elements originally contained in each bucket after the execution of Frederickson's algorithm. The count vector $C$ should also support rank and select queries efficiently. We store these counts encoded in unary, using a 0-bit to mark the border between every two consecutive buckets. Since a total of at most $S$ candidates need to be stored, the count vector $C$ contains at most $S$ ones. Since we have exactly $S$ buckets, $C$ contains $S-1$ zeros. The count vector thus uses $\Theta(S)$ bits. In addition, we create and maintain a wavelet stack $H$-an element hierarchy where each bit corresponds to an element among those whose values fall in the range of the filters. Since there are at most $S$ such elements, the wavelet stack $H$ uses $O(S)$ bits as well. While our algorithm is in action, the wavelet stack is to be updated to indicate the elements that are currently surviving the pruning phases.

We can now iterate efficiently through the active elements. Let $i-1$ be the rank of the element that has just been considered in our iterative scan within the currently active elements. First, we find the index $j$ of the next element to be considered within the wavelet stack. For that we compute

$$
j=H . i n d e x(i),
$$

which is the index of the element we are looking for with respect to those falling between the two filters inherited from Fredrickson's algorithm.

The position $a$ of this element in the count vector $C$ is

$$
a=C \cdot \operatorname{select}(j) \text {. }
$$

The difference between the position of a bit in the count vector, $C$, and the rank of that bit plus one is the index of the bucket that contains the corresponding element. We compute the index $u$ of the bucket containing this element as

$$
u=a-j+1 .
$$

If $u>1$, we calculate the index $z$ that corresponds to the 0-bit resembling the border between the $(u-1)$-th and $u$-th buckets in $C$. This is done by utilizing, $\bar{C}$, the complement vector of $C$ to get

$$
z=\bar{C} \cdot \operatorname{select}(u-1) .
$$

We finally determine the position $g$ of the sought element among Frederickson's candidates within the $u$-th bucket as

$$
g= \begin{cases}a & \text { if } u=1 \\ a-z & \text { if } u>1\end{cases}
$$

If the preceding alive element in the scan was also from bucket $u$, we continue scanning the elements of the $u$-th bucket from where we stopped. Otherwise, we jump to the beginning of the $u$-th bucket, i.e. to the element whose index is $(u-1) \cdot\lceil N / S\rceil+1$ in the input array. We sequentially scan the elements of this 
bucket, discard the ones falling outside the filters and count the others, until locating the $g$-th element among them; this is the one we are looking for.

We can now proceed as in the $\Theta(N)$-bit solution. Starting with the elements surviving Frederickson's algorithm, we recursively determine the medianof-medians and use it to perform the pruning. During this process, we keep the wavelet stack up to date as before. The pruning process continues until only one bucket containing active elements remains, at such point only $O(N / S)$ elements are active. Since this branch of the algorithm is employed only when $S=\Omega(\sqrt{N \lg N})$, the indices of the active elements can fit in the allowable workspace, each in $O(\lg N)$ bits, and we continue the selection in linear time.

Since we are operating on buckets, we might have to spend $\Theta(N / S)$ time for scanning per bucket. However, we note that initially there is at most $S$ candidates and accordingly at most $S$ buckets. Since we prune a constant fraction of the candidates in each iteration, we also reduce the bound on the number of the remaining buckets (those having at least one active element each) by the same constant fraction. Because we skip the buckets that have no active elements, the work done per pass to iterate over the buckets that have at least one active element can be bounded, as elaborated in the next lemma.

Lemma 5.2. Given a read-only input array with $N$ elements, and two filters, such that at most $S$ elements lie in the range of the filters. If $S=\Omega(\sqrt{N \lg N})$, we can solve the selection problem in $O(N)$ time.

Proof: In each pruning iteration we spend time proportional to the number of buckets remaining, while scanning the elements in these buckets and comparing them with the filters. The number of active elements before we apply the $i$-th pruning iteration of the median-of-medians algorithm is at most $S / \mathrm{c}^{i-1}$, for some constant $c>1$. Obviously, the number of buckets that have active elements cannot exceed the number of elements. It follows that, throughout all the passes of the algorithm, the number of scanned buckets is at most $O\left(\sum_{i \geq 1} S / c^{i-1}\right)=$ $O(S)$. Accordingly, the overall work done in scanning these buckets is $O(N)$. Once we have $O(N / S)$ elements remaining, as $S=\Omega(\sqrt{N \lg N})$, we can continue the selection process in the available workspace in $O(N / S)$ time.

The main result of this paper is summarized in the upcoming theorem.

Theorem 5.1. Given a read-only array of $N$ elements and a workspace of $\Theta(S)$ bits where $\lg ^{3} N \leq S \leq N$, it is possible to solve the selection problem in $O\left(N \lg ^{*}(N / S)+N(\lg N) / \lg S\right)$ worst-case time in the space-restricted random-access model.

Theorem 5.1 implies that, in the read-only space-restricted setting, Chan's lower bound 4 for selection in the multi-pass streaming model does not apply to the random-access model.

\section{Conclusions}

We showed that, given an array of $N$ elements in a read-only memory, the $k$-th smallest element can be found in $\Theta(N)$ worst-case time using $\Theta(N)$ bits 
of extra space. We also generalized our algorithm to run in $O\left(N \lg ^{*}(N / S)+\right.$ $N(\lg N) / \lg S)$ time using workspace of $\Theta(S)$ bits, $\lg ^{3} N \leq S \leq N$. Our main purpose was to show that the lower bound proved by Chan 4 for the multi-pass streaming model can be surpassed in the space-restricted random-access model.

In the read-only setting, the selection problem has been studied since 1980 [16. In contrast to sorting, the exact complexity of selection is still open. The time-space trade-off for sorting is known to be $\Theta\left(N^{2} / S+N \lg S\right)$ [2, 19], where $S$ is the size of the workspace in bits, $\lg N \leq S \leq N / \lg N$. The optimal bound for sorting can even be realized using a natural priority-queue-based algorithm [1.

Subsequent to our work, Chan et al. [5] considered the selection problem in the space-restricted random-access model when the elements of the input array are integers, and gave "improved" bounds for this case. Chan et al. 6 also considered the selection (and also sorting) problem in another model, called the restore model, where the input array is allowed to be modified during the process of answering a query, but after the query is answered it has to be restored to its original state. They used the result of Theorem 4.1, and obtained a lineartime selection algorithm with logarithmic amount of extra space. The selection problem in the restore model has also been previously considered by Katajainen and Pasanen [14, who gave a linear-time algorithm that uses a linear number of extra bits for the case when the elements are indivisible (i.e. they can only be compared). Settling the exact complexity of the selection problem in different computational models is still an interesting, partially open problem.

\section{References}

[1] T. Asano, A. Elmasry, and J. Katajainen. Priority queues and sorting for read-only data. In Proceedings of the 10th Theory and Applications of Models of Computation, volume 7876 of Lecture Notes in Computer Science, pages 32-41. Springer-Verlag, 2013.

[2] P. Beame. A general sequential time-space tradeoff for finding unique elements. SIAM Journal on Computing, 20(2):270-277, 1991.

[3] M. Blum, R. W. Floyd, V. Pratt, R. L. Rivest, and R. E. Tarjan. Time bounds for selection. Journal of Computer and System Sciences, 7(4):448461, 1973.

[4] T. M. Chan. Comparison-based time-space lower bounds for selection. ACM Transactions on Algorithms, 6(2):Article 26, 2010.

[5] T. M. Chan, J. I. Munro, and V. Raman. Faster, space-efficient selection algorithms in read-only memory for integers. In Proceedings of the 24 th International Symposium on Algorithms and Computation, volume 8283 of Lecture Notes in Computer Science, pages 405-412. Springer-Verlag, 2013.

[6] T. M. Chan, J. I. Munro, and V. Raman. Selection and sorting in the "restore" model. In Proceedings of the 25th Annual ACM-SIAM Symposium on Discrete Algorithms, pages 995-1004. SIAM, 2014. 
[7] B. Chazelle. A functional approach to data structures and its use in multidimensional searching. SIAM Journal on Computing, 17(3):427-462, 1988.

[8] D. Clark. Compact Pat Trees. PhD thesis, Department of Computer Science, University of Waterloo, 1996.

[9] T. H. Cormen, C. E. Leiserson, R. L. Rivest, and C. Stein. Introduction to Algorithms. The MIT Press, 3rd edition, 2009.

[10] G. N. Frederickson. Upper bounds for time-space trade-offs in sorting and selection. Journal of Computer and System Sciences, 34(1):19-26, 1987.

[11] A. Golynski. Optimal lower bounds for rank and select indexes. Theoretical Computer Science, 387(3):348-359, 2007.

[12] R. Grossi, A. Gupta, and J. S. Vitter. High-order entropy-compressed text indexes. In Proceedings of the 14th Annual ACM-SIAM Symposium on Discrete Algorithms, pages 841-850. SIAM, 2003.

[13] G. Jacobson. Space-efficient static trees and graphs. In Proceedings of the 30th Annual Symposium on Foundations of Computer Science, pages 549-554. IEEE, 1989.

[14] J. Katajainen and T. Pasanen. Sorting multisets stably in minimum space. Acta Informatica, 31(4):301-313, 1994.

[15] J. I. Munro. Tables. In Proceedings of the 16th Conference on Foundations of Software Technology and Theoretical Computer Science, volume 1180 of Lecture Notes in Computer Science, pages 37-42. Springer-Verlag, 1996.

[16] J. I. Munro and M. S. Paterson. Selection and sorting with limited storage. Theoretical Computer Science, 12(3):315-323, 1980.

[17] J. I. Munro and V. Raman. Selection from read-only memory and sorting with minimum data movement. Theoretical Computer Science, 165(2):311$323,1996$.

[18] G. Navarro. Wavelet trees for all. In Proceedings of the 23rd Annual Symposium on Combinatorial Pattern Matching, volume 7354 of Lecture Notes in Computer Science, pages 2-26. Springer-Verlag, 2012.

[19] J. Pagter and T. Rauhe. Optimal time-space trade-offs for sorting. In Proceedings of the 39th Annual Symposium on Foundations of Computer Science, pages 264-268. IEEE, 1998.

[20] R. Raman, V. Raman, and S. R. Satti. Succinct indexable dictionaries with applications to encoding $k$-ary trees, prefix sums and multisets. $A C M$ Transactions on Algorithms, 3(4):Article 43, 2007.

[21] V. Raman and S. Ramnath. Improved upper bounds for time-space tradeoffs for selection. Nordic Journal of Computing, 6(2):162-180, 1999. 